\title{
Ocular Enlargement Following Infantile Corneal Opacification
}

\author{
J. M. TWOMEY, A. GILVARRY, M. RESTORI, C. M. KIRKNESS, A. T. MOORE, \\ A. L. HOLDEN \\ London
}

\begin{abstract}
Summary
Congenital hereditary endothelial dystrophy (CHED) is not generally thought to be associated with other ocular abnormalities. Ultrasonography in a series of twenty eyes (ten patients) with CHED shows ocular enlargement similar to that occurring in uncomplicated axial myopia. There was an inverse relationship between the degree of enlargement and the visual acuity or visual result following penetrating keratoplasty suggesting that infantile corneal oedema sufficient to cause stimulus deprivation may result in abnormal enlargement of the globe.
\end{abstract}

Congenital hereditary endothelial dystrophy (CHED) was first described by Maumenee in $1960{ }^{1}$ Autosomal dominant and recessive patterns of inheritance have been described and the primary defect appears to be an endothelial dysfunction with the secretion of an anomalous Descement's membrane. ${ }^{2}$ Clinically these children present with corneal oedema causing corneal clouding of varying severity and are often misdiagnosed as having congenital glaucoma. The relationship of congenital hereditary endothelial dystrophy with posterior polymorphous dystrophy ${ }^{3}$ and other ocular disorders of neural crest tissue ${ }^{4}$ is unclear.

Wiesel and Raviola demonstrated in $1977^{5}$ that visual deprivation by means of lid sutures in young macaque monkeys led to an increase in the axial length of their occluded eyes. Although the mechanism remains in doubt, it seems that elongation of the eye can be induced by alteration in visual input and is mediated by neural mechanisms. ${ }^{6}$ This suggests that, in addition to genetic factors, environmental influences may be relevant in the development of at least some myopias in animals. Gee and Tabarra have demonstrated $^{7}$ that bilateral and unilateral dense corneal scars of varying aetiology were associated with an increase in the axial length of these eyes compared to control or fellow eyes respectively and in another study anisometropic myopia has been shown to develop in infants with vitreous haemorrhage. ${ }^{8}$

Since corneal oedema may result in a poor visual image from early infancy, CHED may prove to be a good model for studying the effects of early visual deprivation on ocular growth in the human eye.

It has been suggested that axial length measurements may be useful in the diagnosis and management of patients with congenital glaucoma. ${ }^{9}$ Congenital hereditary corneal endothelial dystrophy may be misdiagnosed initially as congenital glaucoma ${ }^{2}$ despite normal corneal diameters and intra-ocular pressure. If these eyes also showed ocular enlargement there would be further grounds

From Corneal Clinic, Moorfields Eye Hospital, City Road, London EC1 and Institute of Ophthalmology, Judd Street, WC1.

Correspondence to: A. Gilvarry, MRCPI, FRCSI, FCOphth., Moorfields Eye Hospital, City Road, London EC1V 2PD. 
for caution. In an effort to document ocular size and to assess the possible influence of early infantile corneal clouding on ocular development we reviewed all patients currently under the care of the corneal clinic at Moorfields Eye Hospital known to have CHED in which a clinical and pathological diagnosis had been made.

\section{Patients and Methods}

It is possible to obtain an accurate outline of the globe on B-mode ultrasonography and in addition to the axial length we were able to make estimates of the sagittal and transverse diameters and calculations of ocular volume.

The axial length was first measured from anterior cornea to vitreo-retinal interface on the A-mode and this measurement was then used to find the central B-mode section using the lens and optic nerve as landmarks. We assumed elliptical saggital and transverse cross-sections of the globe and used the ellipse facility on the scan to conform to external ocular shape (Fig. 1). The volume was calculated from the equation $\mathrm{V}=4 / 3 \pi \mathrm{abc}$ where $\mathrm{a}$ is the antero-posterior semi-axis averaged over sagittal and transverse plane ellipses and, b and $c$ are the sagittal and transverse semi-axes of those respective planes.

Four eyebank eyes were examined to correlate ultrasound volume estimates with those obtained from the fluid displacement of these same eyes. There was a discrepancy of less than $10 \%$ between these two measurements.

We measured twenty eyes in ten patients with congenital hereditary endothelial dystrophy; sixteen eyes had undergone at least one penetrating keratoplasty. No patient was

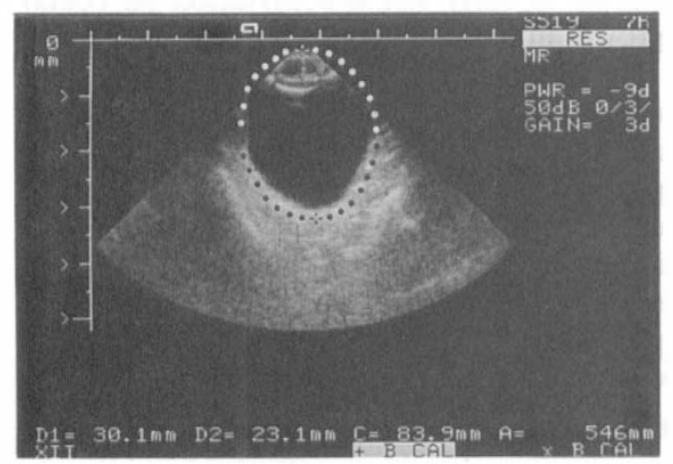

Fig. 1 Outline of glo be using the ellipse facility on the $B$ mode ultrasound. thought to have a cranio-facial disorder and any patients with a history of raised intraocular pressure was excluded.

Nine patients were thought to have the autosomal dominant variant of the disease and one the recessive form. The age of onset of visual problems ranged from infancy to 15 years of age and the age at examination was between 16 and 67 years of age.

For the purposes of comparison these twenty eyes were divided into two groups; those with an acuity of $6 / 24$ or better and those in whom the best recorded visual acuity either before or after keratoplasty was $6 / 36$ or worse. There were nine eyes in the first, good acuity group and eleven in the second or poor acuity group.

Stastistical comparison between these groups of eyes was by means of a students ' $t$ ' test.

Ultrasound measurements were also recorded from a control group of emmetropic patients recorded as seeing 6/9 or better and from a group of myopic patients (greater than $-6.00 \mathrm{D})$ with a corrected acuity of $6 / 12$ or better.

\section{Results}

Axial length and visual acuity (Fig. 2). The best recorded visual acuity is plotted as a function of axial length. It can be seen that the axial length of eyes with good vision $(6 / 24$ or better) is different from those eyes with poor visual acuity (6/36 or worse).

Eyes with good acuity show a range of axial lengths similar to that of the emmetropic control eyes. Eyes with poor acuity show considerably greater axial lengths. This difference is statistically significant $(0.01>p>0.001)$ despite two eyes $(a$ and $b)$ not conforming to this pattern.

Ocular volume and visual acuity (Fig. 3). The best recorded visual acuity is plotted as a function of ocular volume. A similar separation of these two groups is seen to occur; those eyes with good acuity showing a range of ocular volumes similar to the emmetropic control group whilst those eyes with poor acuity have a significantly larger ocular volume $(0.05>p>0.02)$. Two eyes ( $a$ and $b)$ are exceptions again in that they have poor acuity and are small. 
CONTROLS

GOOD ACUITY GROUP
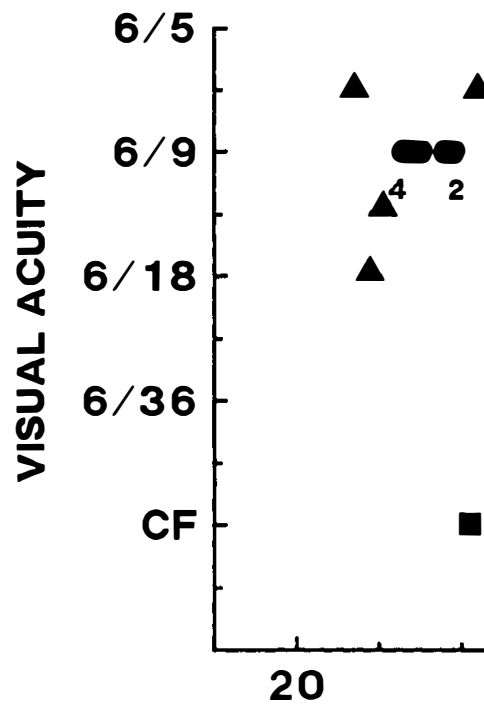

POOR ACUITY GROUP

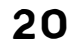

25

30

AXIAL LENGTH in $\mathrm{mms}$

Fig 2 Visual acuity v. axial length. CHED patients (good acuity 6/24 or better(, (poor acuity 6/36 or worse) and emmetropic controls (6/9).

During the measurements a tendency for asymmetrical ocular enlargement with the horizontal or transverse diameters outstripping the vertical or sagittal diameters in the larger eyes was noted. The two scans (Figs $4 a$ and $4 b$ ) are of the same eye; the transverse section is seen to be of a greater diameter than the sagittal or vertical section. The axial length measurements are identical.

Ratio of transverse and sagittal diameters and visual acuity. On comparing the ratio of transverse to sagittal diameters with visual acuity a similar separation of the two groups is apparent as shown in Fig. $5(0.05>\mathrm{p}>0.02)$.

Those patients with good acuity have smaller eyes whilst those with the poorer acuity have large globes with an asymetrical pattern of ocular enlargement.

\section{Discussion}

The inability reliably to distinguish between the various forms of myopia; physiological, pathological and those associated with ocular or systemic disease has made the interpretation of experimental models and clinical observations difficult. Patterns of growth in pathologically myopic eyes may not be applicable to lower and probably physiological degrees of myopia.

In addition to axial length; corneal curvature, anterior chamber depth and lens thickness all contribute to the refractive status of an individual. The major components are length and corneal curvature; lens thickness and anterior chamber depth being minor factors in the final refractive outcome once ocular growth is completed. Curtin ${ }^{10}$ notes that the relative contribution of axial length to a final myopic refraction increases with increasing degrees of myopia. It was not possible to assess any tendency towards 'emmetropisation' (i.e. corneal flattening in association with increasing axial length) in our group of eyes as many patients had undergone penetrating keratoplasty. For the same reason our measurements were confined to ocular dimensions rather than refraction as the pres- 
CONTROLS

GOOD ACUITY GROUP

POOR ACUITY GROUP

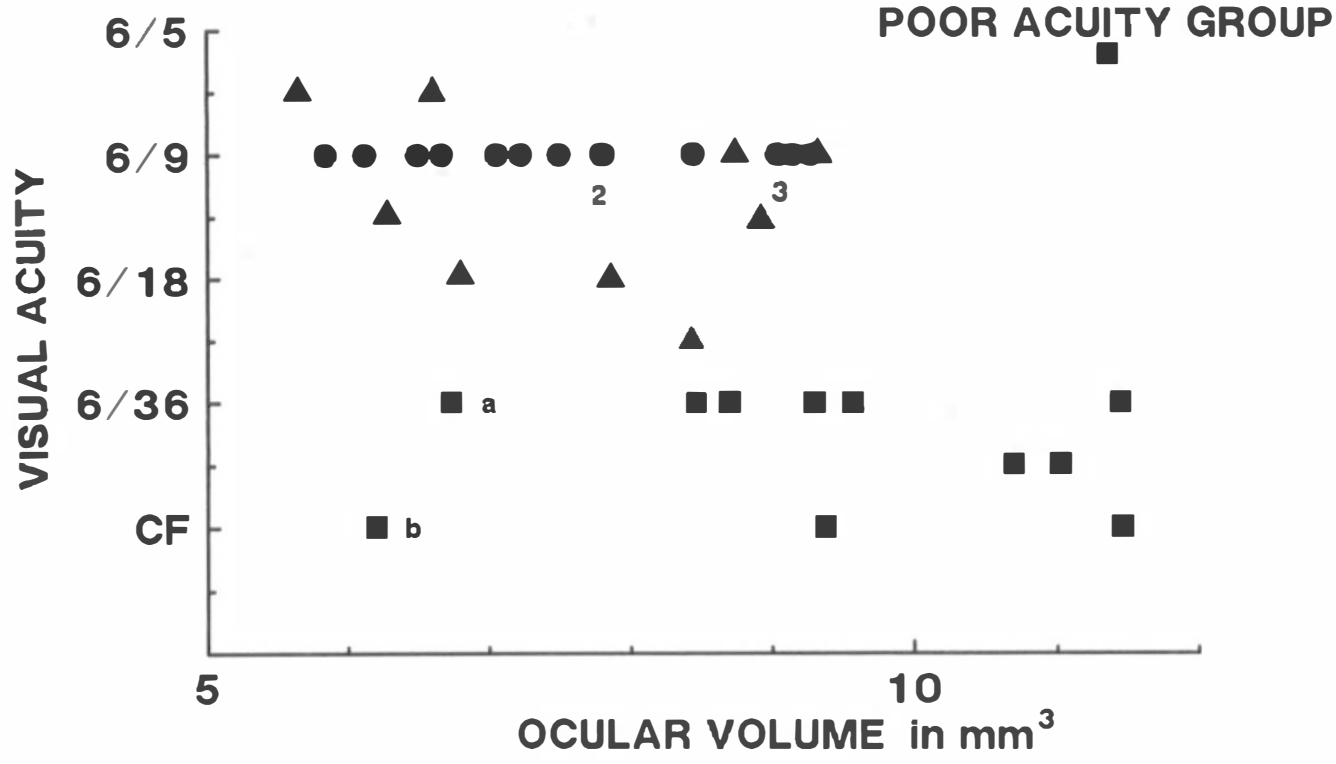

Fig. 3 Visual acuity v. ocular volume. CHED patients (good acuity 6/24 or better), (poor acuity 6/36 or worse) and emmetropic controls (6/9).

ence of a large number of corneal grafts rendered the assessment of the contribution of corneal curvature to refraction unreliable.

Our results indicate that in this disorder varying severity of corneal clouding and form visual deprivation in early infancy is associated with varying degrees of ocular enlargement. There is a clear relationship between the severity of the amblyopia and the degree of ocular enlargement suggesting that the

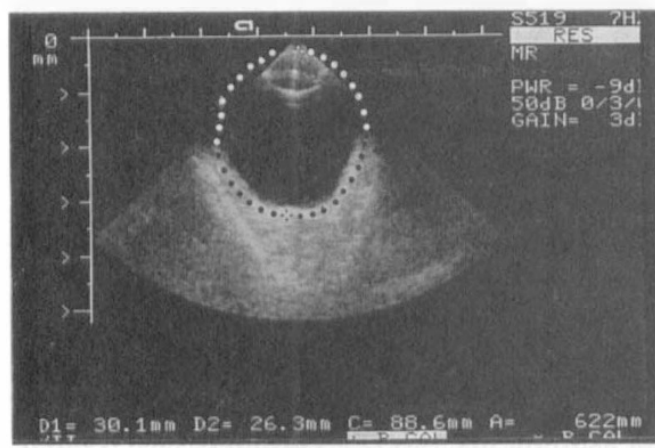

Fig. 4a B scan or large eye. Transverse section. enlargement is secondary and due to a failure of emmetropisation rather than merely an associated feature of the disease.

The pattern of ocular enlargement, with an increase in the ratio of transverse to sagittal diameters is similar to that in myopes generally and which was demonstrated by Dellar, O'Connor and Sorsby in a study of 45 myopic eyes in 1947 and cited by Curtin. ${ }^{10}$ This was confirmed in our comparison of the

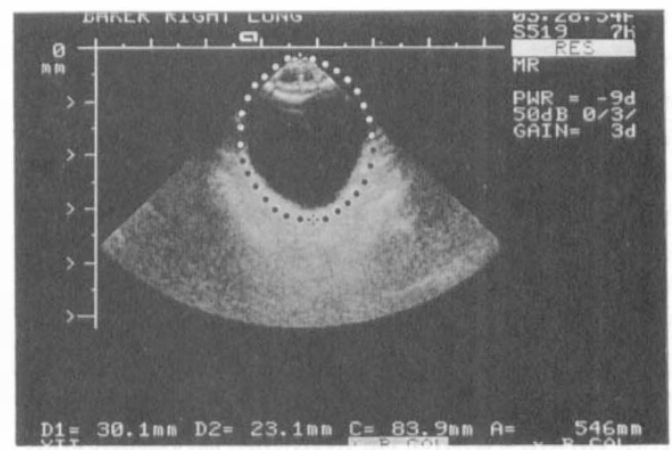

Fig. 4b B scan of same eye. Sagittal section. 
CONTROLS

GOOD ACUITY GROUP

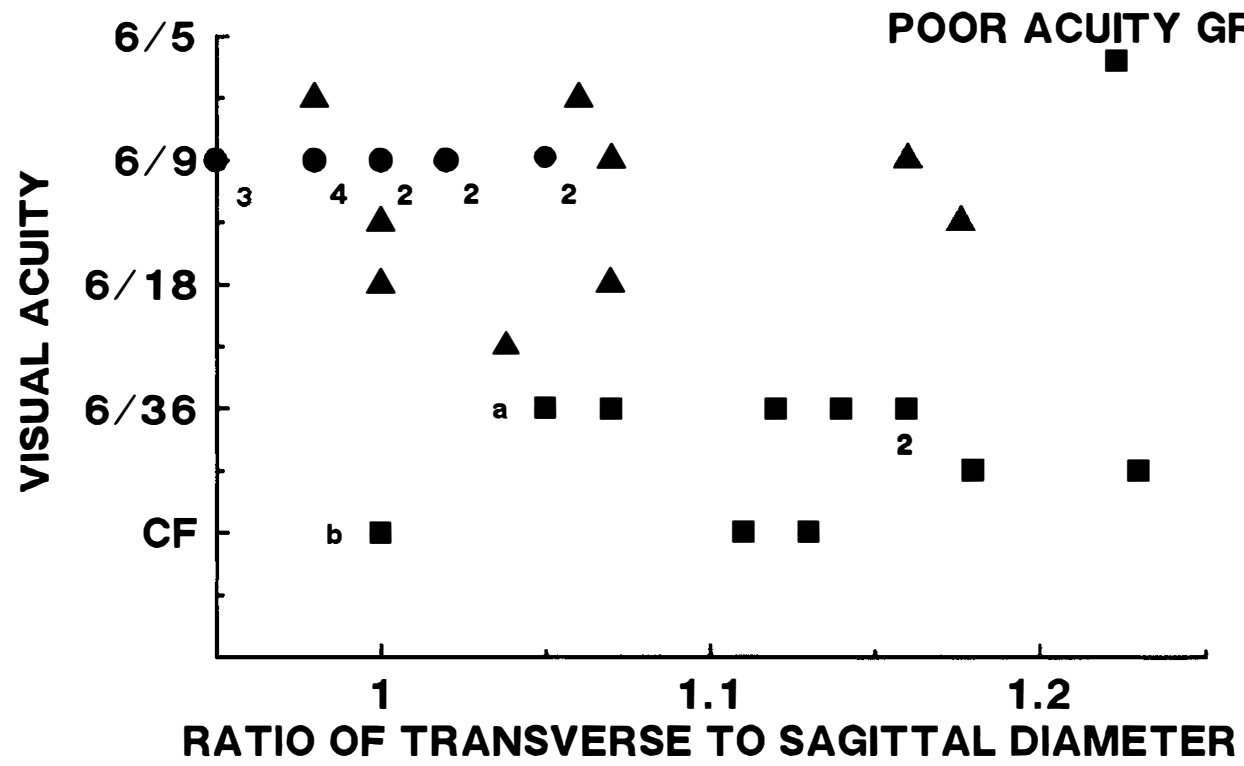

Fig. 5 Visual acuity v. transverse/sagittal diameter ratio. CHED patients (good acuity 6/24 or better), (poor acuity $6 / 36$ or worse) and emmetropic controls (6/9).

ratio of transverse to sagittal diameters in the two control groups; myopes (greater than $-6.00 \mathrm{D}$ ) and emmetropes (Fig. 6). The histogram demonstrates this separation $(0.01>\mathrm{p}>0.001)$.

Two eyes ( $a$ and $b$ ), however, have poor acuity and are small. These eyes are the fellow eyes of those with good acuity in patients with clinically symmetrical corneal dystrophy. Both these eyes were noted to be esotropic in infancy and our measurements might support the suggestion that fixation and, therefore, form vision are associated with a shift to myopia. In patients without strabismus who had purely stimulus deprivation, there was a clear relationship between the severity of the amblyopia and the enlargement of the eye. In these two eyes the amblyopia appears related to strabismus rather than stimulus deprivation and was not associated with ocular enlargement. Fixation and presumed macular retinal image blue appears necessary to induce the ocular enlargement, implying some 'attempt' to achieve clear vision.
Our results showing the varying effect of corneal opacity in this disorder on ocular growth, presumably a consequence of varying severity of corneal oedema, support the idea of a strong environmental influence in the genesis of at least some myopias in man as has been demonstrated in animal models.

These patients show a similar response to stimulus deprivation as has been shown to occur in various animal studies. In the lidsuture model of myopia, Raviola and Wiesel ${ }^{6}$ demonstrated the enlargement to be independent of an intact visual cortex. The role of accommodation in inducing this growth is uncertain, there being considerable interspecies variation. Holden et al ${ }^{11}$ have shown that in chicks a local myopia may be induced in response to occlusion along a single axis, affecting a part of the retina only. These findings suggest that the mechanism inducing the increase in axial legnth occurs at a local level (i.e. within the eye) in response to retinal stimulus deprivation. Blockage of ganglion cell action potentials in the Tree Shrew ${ }^{12}$ failed 


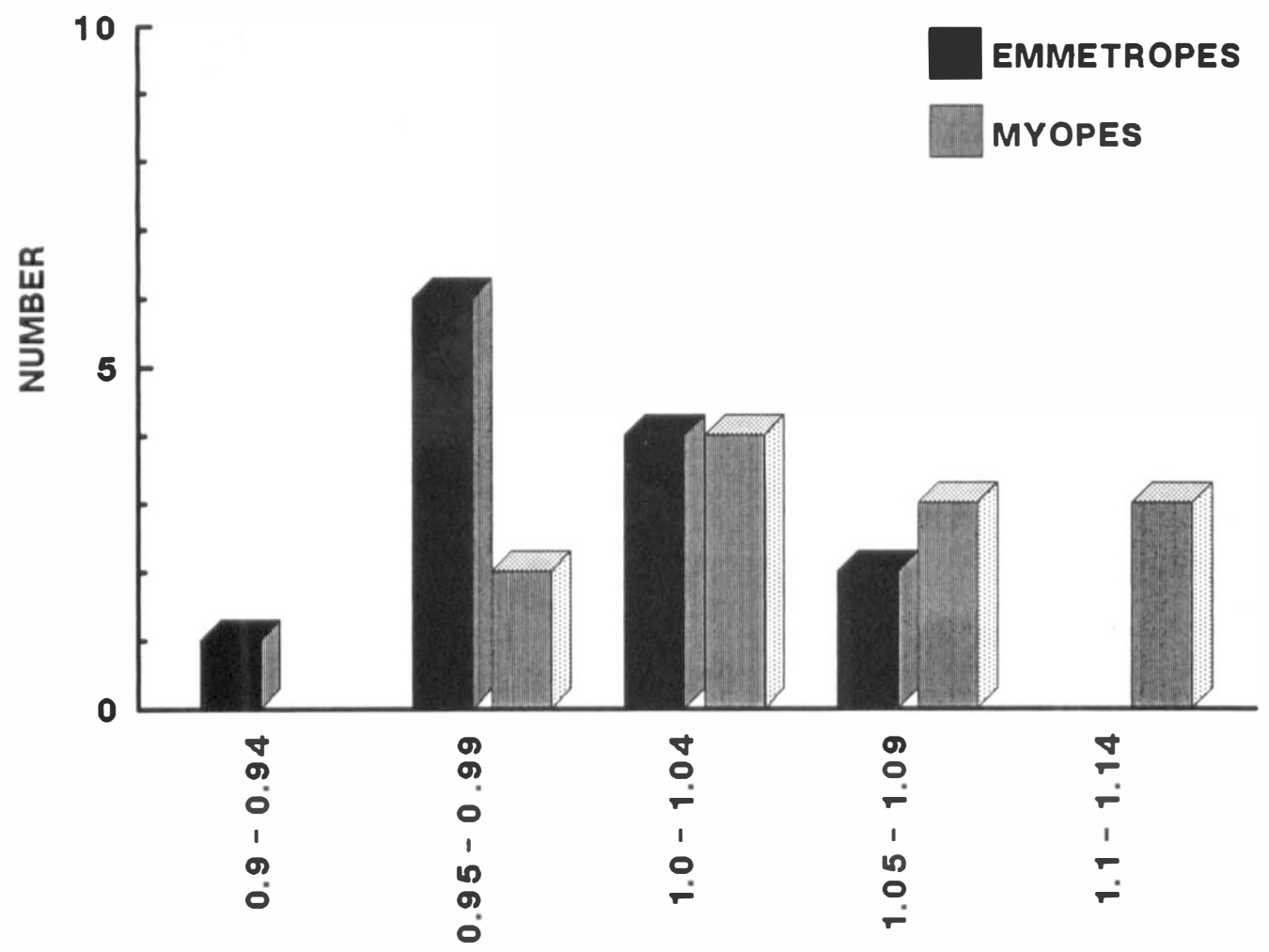

\section{RATIO OF TRANSVERSE TO SAGITTAL DIAMETER}

Fig. 6 Histogram comparing the ratio of transverse to sagittal diameters in emmetropes and myopes (greater than $-6.00 D)$.

to block the ocular enlargement in response to monocular visual deprivation and retinal dopamine has been suggested to pay a role in the link between visual experience and postnatal ocular growth in the chick. ${ }^{13}$

The similarity in growth pattern between these patients and myopes generally, with the asymmetrical pattern of ocular growth, might indicate that genetic and environmental controls act via a final common and possibly local pathway.

From the clinical standpoint these findings might allow one to be more confident in predictions of benefit likely to accrue from penetrating keratoplasty in patients with early onset corneal opacity and our results would also caution against excessive reliance on measurements of ocular size in the diagnosis and management of congenital glaucoma, the most important differential diagnosis.

We should like to thank Mr A. D. McG. Steele, Mr N. S. C. Rice and Mr J. P. Lee for permission to report their cases.

\section{References}

${ }^{1}$ Maumenee AE: Congenital hereditary corneal dystrophy. Am J Ophthalmol 1960, 50: 1114-24.

${ }^{2}$ Kirkness CM, McCartney A, Rice NSC, Garner A, Steele ADMcG: Congenital hereditary corneal oedema of Maumenee: its clinical features, management and pathology. Br J Ophthmol 1987, 71: $130-44$.

${ }^{3}$ McCartney ACE and Kirkness CM: Comparison between posterior polymorphous dystrophy and congenital hereditary endothelial dystrophy of the cornea. Eye 1988, 2: 63-70. 
${ }^{4}$ Bahn CF, Falls HF, Varley GA, Meyer RF, Edelhauser HF, Bourne WM: Classification of corneal endotheliál disorders based on neural crest origin. Ophthalmology 1984, 91: 558-63.

${ }^{5}$ Wiesel TN and Raviola E: Myopia and eye enlargement after neonatal lid fusion in monkeys. Nature 1977, 266: 66-8.

${ }^{6}$ Raviola E and Wiesel TN: An animal model of myopia. New Eng J Med 1985, 312: 1609-15.

${ }^{7}$ Gee SS and Tabarra KF: Increase in ocular axial length in patients with corneal opacification. Ophthalmology 1988, 95: 1276-8.

${ }^{8}$ Miller-Meeks M, Bennett SR, Keech RV: Development of anisometropic myopia in infants with vitreous haemorrhage. Invest Ophthalmol Vis Sci 1989, 30 (3-suppl): 302.
${ }^{9}$ Sampaolesi R and Caruso R: Ocular echometry in the diagnosis of congenital glaucoma. Arch Ophthalmol 1982, 100: 574-7.

${ }^{10}$ Curtin BJ: The Myopias: Basic science and clinical management. Philadelphia, Harper and Row, 1985.

${ }^{11}$ Holden AL, Hodos W, Hayes B P, Fitzke FW: Myopia: Induced normal and clinical. Eye 1988, 2 (suppl): S242-56.

${ }^{12}$ Norton TT, Essinger JA, McBrien NA: Lid-suture myopia in Tree Shrew despite blockade of ganglion cell action potentials. Invest Ophthalmol Vis Sci 1989, 30 (3-suppl): 31.

${ }^{13}$ Stone RA, Lin T, Laties AM, Iuvone PM: Retinal dopamine and form deprivation myopia. Proc Natl Acad Sci USA 1989, 86: 704-6. 